\title{
Influence of Oscillation Frequency and Nonlinear Structural Material of Airfoil in Wind Turbine System During Dynamic Stall
}

\author{
Cheng-Chi Wang $^{1 *}$ and Aniket Mishra ${ }^{2}$ \\ ${ }^{1}$ Graduate Institute of Precision Manufacturing, National Chin-Yi University of Technology, \\ No. 57, Sec. 2, Zhongshan Rd., Taiping Dist., Taichung 411030, Taiwan \\ ${ }^{2}$ Department of Mechanical Engineering, National Chin-Yi University of Technology, \\ No. 57, Sec. 2, Zhongshan Rd., Taiping Dist., Taichung 411030, Taiwan
}

(Received May 24, 2021; accepted September 28, 2021)

Keywords: bifurcation, structural nonlinearity, reduced frequency of oscillation, chaos

We study the nonlinear behavior of a wind turbine airfoil oscillating along the pitch mode of oscillation. In the analysis, the nonlinearity of the structural material, the airspeed, and the reduced frequency of oscillation are the important parameters considered. The equation of motion for the pitching oscillation case is modeled and derived using the Office National d'Etudes et de Recherches Aérospatiales (ONERA) dynamic stall model. In the ONERA dynamic stall model, the aerodynamic loads are given in terms of differential equations. We focus on the influence of the material structural nonlinearity and nondimensional airspeed on the wind turbine airfoil during the pitch mode of oscillation. The type of airfoil considered is the National Advisory Committee for Aeronautics (NACA) 0012 airfoil. To understand the effects of dynamic stall on the system under different situations of structural nonlinearity, we use bifurcation plots, phase plots, Poincaré maps, and the maximum Lyapunov exponent (MLE) to analyze the behavior of the airfoil and avoid the effect of non-periodic motion of the airfoil on the wind turbine system. The results show that chaos occurs for specific parameters of the airfoil of the wind turbine, which can be avoided by avoiding certain operating conditions corresponding to chaotic motion. This finding can be used as a guideline for wind turbine design and decrease the cost of maintenance.

\section{Introduction}

With the increasing demand for wind energy due to its renewable nature, wind energy has much greater importance than a few decades ago. Hence, wind turbines are now being used by many countries to produce energy. Because of this, it is important to build wind turbines with robust designs. Various natural phenomena can damage the wind turbine structure. The most important phenomena studied in this paper are the structural nonlinearity of the airfoil and the dynamic stall phenomenon. The structural nonlinearity of the airfoil material induces nonlinear behavior of the wind turbine and reduces the efficiency of power generation. ${ }^{(1,2)}$ When an airfoil undergoes a rapid change in the angle of attack, dynamic stall occurs. This rapid change in the ${ }^{*}$ Corresponding author: e-mail: wcc@ncut.edu.tw https://doi.org/10.18494/SAM.2021.3625 
angle of attack can be caused by a sudden gust of wind or yaw misalignment. As the dynamic stall is increased, it can cause the airfoil to vibrate in a nonlinear mode and damage the airfoil.

Dynamic stall and the methods to control it have been of interest to many researchers worldwide. Zhong et al. ${ }^{(3)}$ proposed the installation of a small rod in front of the leading edge of a symmetrical airfoil to control the dynamic stall of a vertical-axis wind turbine. This setup helps cancel the flow separation of an airfoil at large angles of attack. In their experiment, a National Advisory Committee for Aeronautics (NACA) 0018 airfoil was analyzed. They experimentally found that, using the rod, the average tangential force and the power coefficient were considerably increased. Zhu et al. ${ }^{(4)}$ analyzed the dynamic stall of a 2D airfoil, 3D nonrotating blade, and 3D rotating blade undergoing sinusoidal pitch oscillations. They found that rotational augmentation delayed the onset of dynamic stall. Huang et al. ${ }^{(5)}$ analyzed the effect of free-stream turbulence on the dynamic stall of wind turbine blades and found that the freestream turbulence in a turbulent flow at a turbulence level of 5\% helped improve the aerodynamic performance of the wind turbine blade. Zhu et al. ${ }^{(6)}$ introduced passive vortex generators (VGs) on wind turbine airfoils to delay the onset of static stall and improve the aerodynamic performance. They experimentally found that the VGs delayed the onset of dynamic stall and also helped to increase the lift coefficient by $40 \%$. Liu et al. ${ }^{(7)}$ used the Beddoes-Leishman (B-L) model to predict unsteady airloads on wind turbine airfoils. By comparing the original B-L model for an S809 type airfoil oscillating in pitch mode for different mean angles of attack, oscillation amplitudes, and reduced frequencies, they found that the modified B-L model showed better performance. Wang and Zhao ${ }^{(8)}$ used a highly efficient optimization method to alleviate the dynamic stall characteristics of a rotor airfoil. They experimentally found that the optimized airfoil had a larger leading edge radius than an OA209 airfoil. The pressure gradient of the optimized airfoil was decreased under dynamic stall. Thus, the aerodynamic loads of the optimized airfoil performed more stably than those of the OA209 airfoil. Niu et al. ${ }^{(9)}$ found a way to control dynamic stall by introducing a variable-droop leading edge (VDLE). The VDLE dramatically decreased the local angle of attack near the leading edge when the overall angle of attack became too large. Zhao et al. ${ }^{(10)}$ numerically investigated the effects of synthetic jet control over a rotor airfoil. They found that a synthetic jet can be the best way to control dynamic stall and its effects when it is located near the flow separation point. Sun and Sheikh ${ }^{(11)}$ used steady and unsteady tangential blowing to suppress the dynamic stall on an oscillating airfoil and found that in the case of tangential blowing, the blowing jet momentum coefficient helped achieve good flow control.

Ekaterinaris et al. ${ }^{(12)}$ devised a method to predict dynamic stall computationally. The prediction of dynamic stall is important for flight vehicles, turbomachinery, and wind turbines. Akbari and Price ${ }^{(13)}$ simulated dynamic stall for an NACA 0012 airfoil using a vortex method. They observed that for pitching oscillation of the airfoil, flow separation occurred at higher incidences than static stall. Larsen et al. ${ }^{(14)}$ presented a model for the aerodynamic lift of wind turbines under dynamic stall. They found that their model for dynamic stall performed well when one state variable was used for trailing edge separation. Liu and Zhang ${ }^{(15)}$ investigated the stall flutter characteristics of a high-aspect-ratio composite wing. They found that geometric nonlinearity had a significant effect on the stall flutter characteristics. Mamouri et al. ${ }^{(16)}$ 
simulated an airfoil oscillating with different frequencies to observe dynamic stall. They found that high entropy was generated by the airfoil, which decreased its efficiency.

Taller wind turbine towers are subjected to higher wind pressures and are more vulnerable to seismic loads. Figure 1 illustrates a wind turbine tower in Goldenstedt, Germany, that collapsed owing to strong wind and another wind turbine tower in Kashima City, Japan, that tilted owing to an earthquake. Since wind turbine structures are subjected to various types of load, it is imperative to understand how wind turbines respond to such loads, and design them in an economic and easy-to-construct way to avoid catastrophic failures. ${ }^{(17)}$

However, there has been little research on the dynamic influence of the nonlinear structural material, airspeed, and oscillation frequency of airfoils during dynamic stall, and it is important to analyze the coupling effect of the oscillation frequency and nonlinear structural material to avoid damage to wind turbine systems.

\section{Analysis Method}

The equation of motion for the airfoil [Eq. (1)] is analyzed and modeled by considering different values of the effective angle of attack $(\alpha)$, nondimensional airspeed $(U)$, reduced frequency of oscillation $\left(K_{1}\right)$, structural nonlinearity $\left(K_{n l}\right)$, amplitude of the force $\left(F_{0}\right)$, mass ratio $(\mu)$, radius of gyration $\left(R_{a}\right)$, aerodynamic moment coefficient $\left(C_{m}\right)$, and nondimensional time $(\tau)$. The reduced frequency of oscillation, nondimensional time, and forcing amplitude are modeled using a sinusoidal wave while the other parameters are modeled as constants.

$$
\alpha^{\prime \prime}=\frac{2 C_{m}}{\pi \mu R_{a}^{2}}+F_{0} \sin \left(K_{1} \tau\right)-\frac{\alpha}{U^{2}}-K_{n l}
$$

The Runge-Kutta method is applied to solve Eq. (1) and the Simulink model of MATLAB is used to simulate the dynamical system. In the Simulink model, the important parameters can be changed and the changes are observed in bifurcation plots, phase plots, and Poincaré maps.

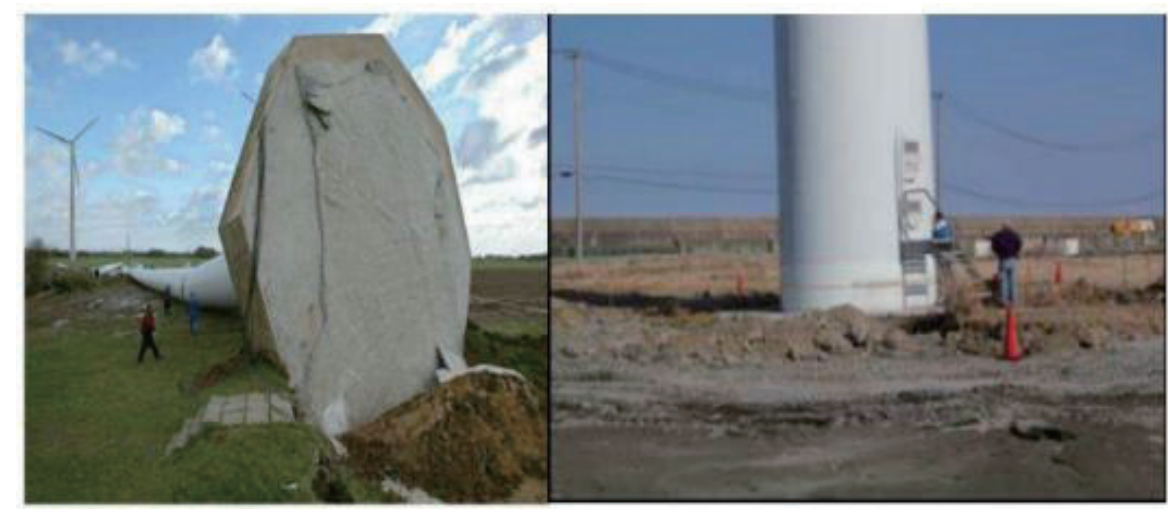

(a)

(b)

Fig. 1. (Color online) Turbines that collapsed in (a) Germany and (b) Japan. ${ }^{(17)}$ 


\section{Results and Discussion}

\subsection{Phase plots and Poincaré maps}

As shown in Fig. 2, when $U=7$ and $K_{n l}=0.01$, the effective angle of attack behaves an irregular manner at a lower frequency of oscillation $\left(K_{1}=0.25,0.6\right)$, and when $K_{1}$ increases to 0.8 , non-periodic motion replaces the regular motion. The motion can be verified by a Poincaré map and it can be calculated from the phase plots. A Poincaré map represents the intersection of points in a dynamic orbit obtained from the phase plots, and is a helpful means of analyzing the stability of systems. A single point in a Poincare map reveals that the motion is T-periodic, a two-point Poincaré map indicates subharmonic motion of period 2T, a closed curve formed by discrete points indicates quasi-periodic motion, and a cluster of closely spaced points indicates chaotic motion. In this way, a Poincaré map is useful to distinguish between various types of motion. ${ }^{(18)}$ In the Poincaré maps in Fig. 3, many discrete points appear and are dispersed in the figure, indicating multi-T subharmonic motion. ${ }^{(18)}$ Thus, when $K_{1}=0.25$ and 0.6 , the system undergoes stable motion as shown in Figs. 3(a) and 3(b), respectively. However, the closed curve formed by discrete points in Fig. 3(c) means that quasi-periodic motion appears when $K_{1}=0.8$.

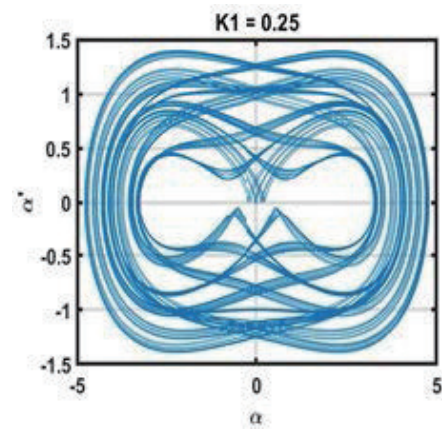

(a)

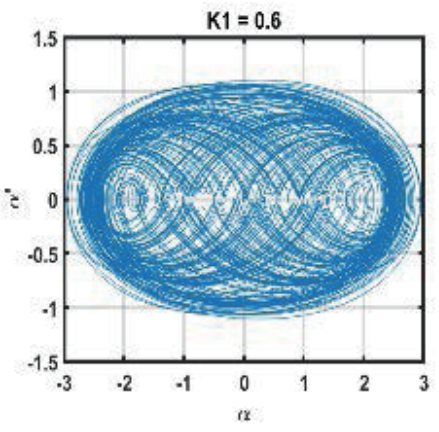

(b)

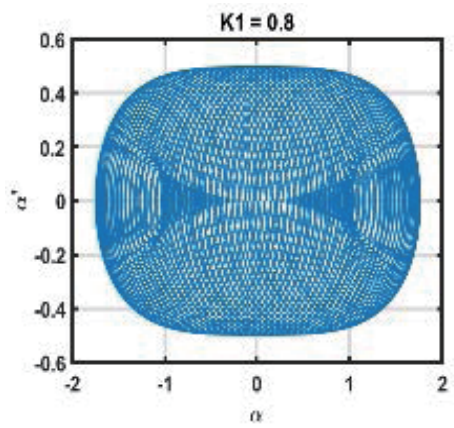

(c)

Fig. 2. (Color online) Phase plots of effective attack angle when $U=7$ and $K_{n l}=0.01$ : (a) $K_{1}=0.25$, (b) $K_{1}=0.6$, and (c) $K_{1}=0.8$.

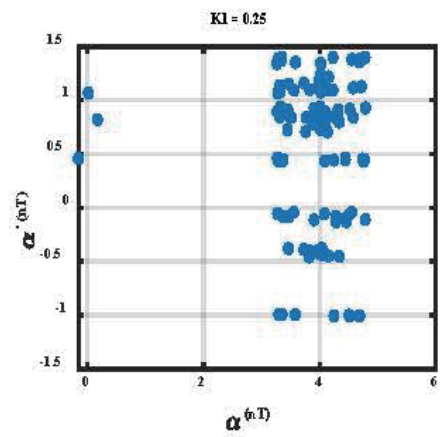

(a)

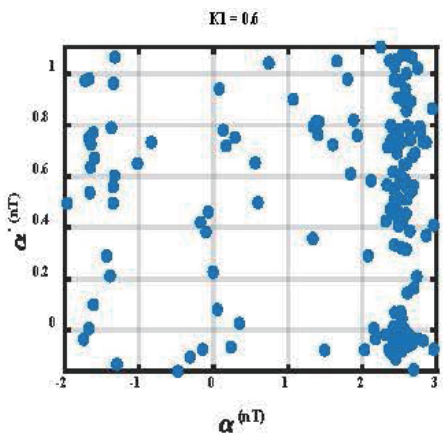

(b)

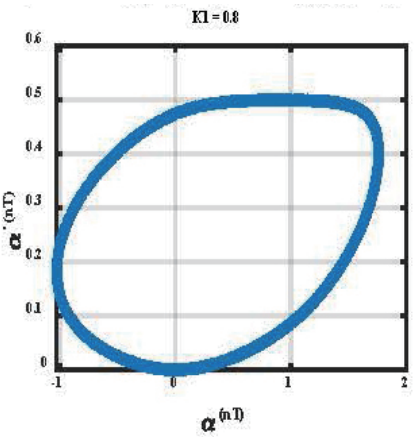

(c)

Fig. 3. (Color online) Poincaré maps of effective attack angle when $U=7$ and $K_{n l}=0.01$ : (a) $K_{1}=0.25$, (b) $K_{1}=0.6$, and (c) $K_{1}=0.8$. 
When $K_{n l}$ is increased to 0.05 , the behavior of the effective angle of attack changes. The phase plots in Fig. 4 show that the system behaves unstably when $K_{1}=0.25,0.6$, and 0.8 . Meanwhile, four clusters appear in the Poincaré map shown in Fig. 5(a), verifying the occurrence of chaos when $K_{1}=0.25$. For $K_{1}=0.6$ and 0.8 , most discrete points are clustered in a specific area as shown in Figs. 5(b) and 5(c), respectively, also verifying the occurrence of chaos. It is thus demonstrated that the system behaves unstably when $K_{n l}$ is increased.

\subsection{Bifurcation diagram and maximum Lyapunov exponent (MLE)}

\subsubsection{Influence of structural nonlinearity on wind turbine system}

As shown in Figs. 6(a) to 9(a), the influence of structural nonlinearity on a wind turbine system is analyzed and the reduced frequency of oscillation is used as an analysis parameter to explore the influence of the oscillation frequency on the system, where the frequency of oscillation is set to between 0.2 and 1 to model actual operating conditions.

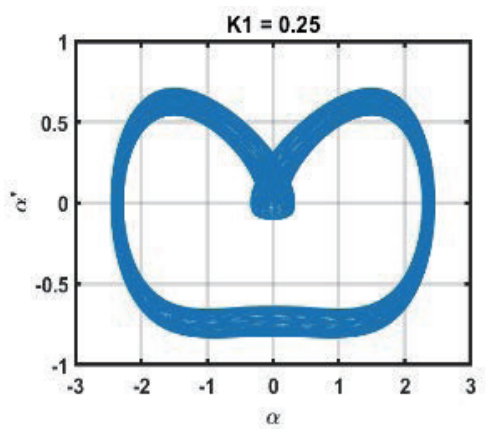

(a)

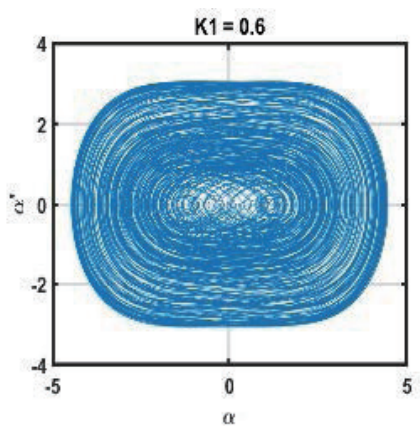

(b)

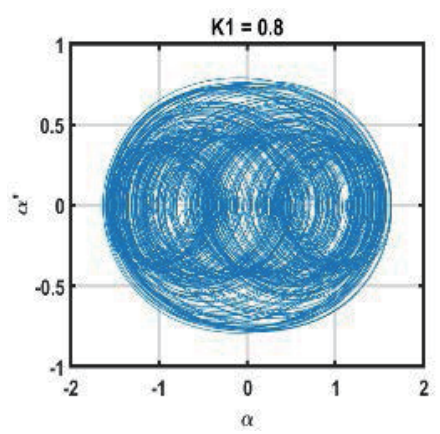

(c)

Fig. 4. (Color online) Phase plots of effective attack angle when $U=7$ and $K_{n l}=0.05$ : (a) $K_{1}=0.25$, (b) $K_{1}=0.6$, and (c) $K_{1}=0.8$.

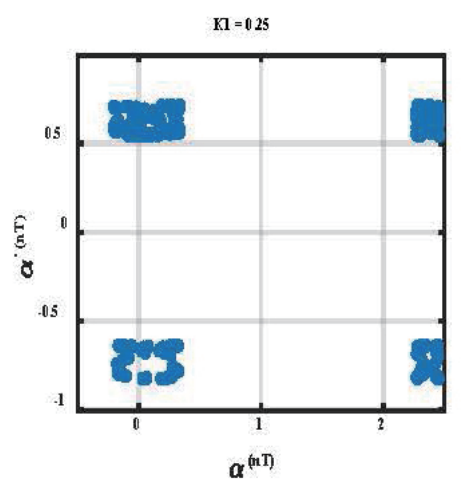

(a)

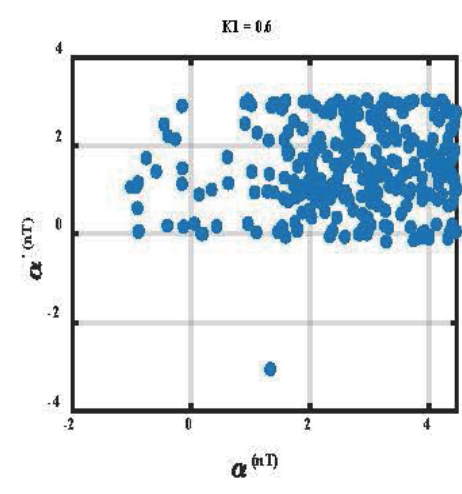

(b)

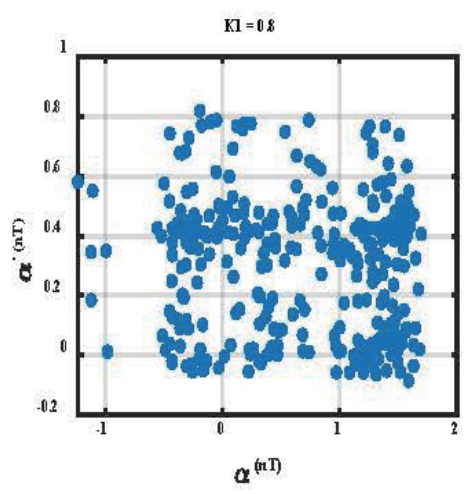

(c)

Fig. 5. (Color online) Poincaré maps of effective attack angle when $U=7$ and $K_{n l}=0.05$ : (a) $K_{1}=0.25$, (b) $K_{1}=0.6$, and (c) $K_{1}=0.8$. 


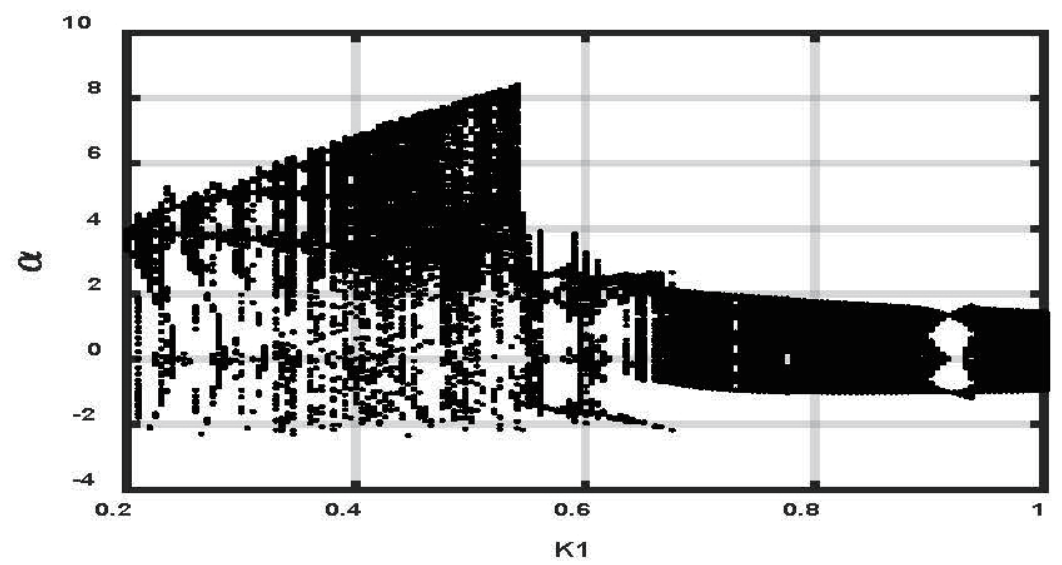

(a)

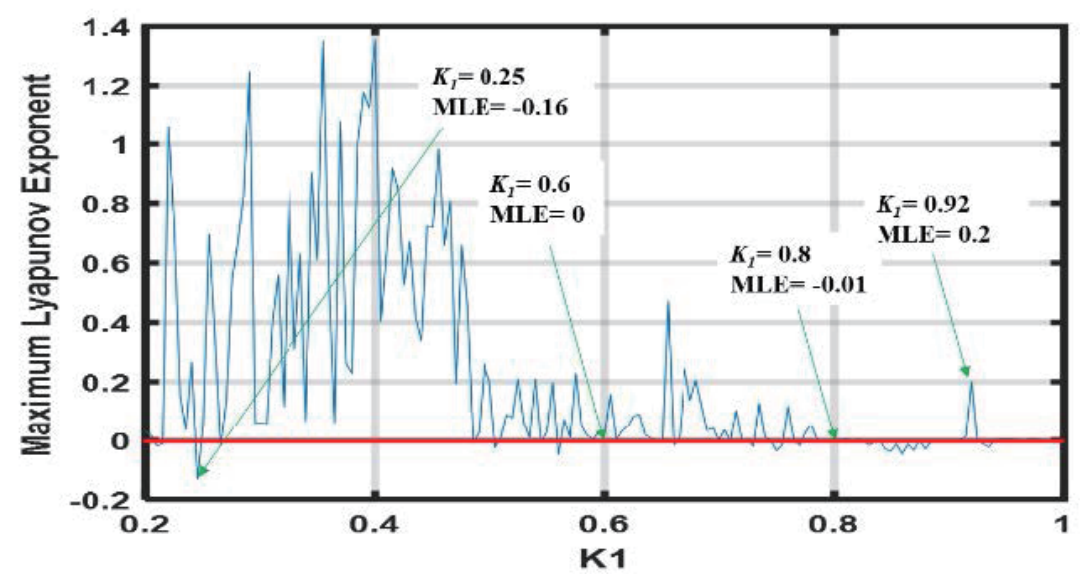

(b)

Fig. 6. (Color online) (a) Bifurcation diagram and (b) MLE for $U=7$ and $K_{n l}=0.01$. Frequency of oscillation is used as an analysis parameter.

To verify the occurrence of chaos, the MLE is used to prove the chaotic behavior of the system as shown in Figs. 6(b) to 9(b). The MLE plot is constructed with the reduced frequency of oscillation and compared with the bifurcation diagram. A positive value of MLE signifies that the motion is chaotic in nature, while a negative or zero value signifies that the motion is periodic, subharmonic, or quasi-periodic.

When $U=7$ and $K_{n l}=0.01$, the effective attack angle is between -2.1 and 8.3 and the system exhibits unstable motion when $K_{1}<0.57$, as shown in Fig. 6(a). However, periodic motion sometimes occurs such as at $K_{1}=0.25$. This phenomenon is confirmed by the Poincaré map shown in Fig. 3(a). When $K_{1}>0.6$, the effective attack angle undergoes quasi-periodic motion between values of 2.0 and -2.0 as shown in Fig. 6(a). This quasi-periodic movement for $K_{1}=0.8$ is verified by the formation of a circle of discrete points in the Poincaré map in Fig. 3(c). The stability of the effective attack angle suggests that the system can be operated within the range of $0.8 \leq K_{1} \leq 1.0$ except for $K_{1}=0.92$. 
From the MLE plot shown in Fig. 6(b), for $U=7$ and $K_{n l}=0.01$, the behavior of the system is non-chaotic at $K_{1}=0.25,0.6$, and 0.8 , for which the values of MLE are $-0.16,0$, and -0.01 , respectively. Moreover, the values of MLE over the range of $0.2 \leq K_{1}<0.6$ are greater than those over the range of $0.6 \leq K_{1} \leq 1.0$. The results of MLE are consistent with the bifurcation diagram.

When $K_{n l}$ is increased to 0.05 under the same $U$, we observe that chaotic behavior occurs more frequently for $K_{n l}=0.05$ than for $K_{n l}=0.01$ in the same range of $0.2 \leq K_{1} \leq 1.0$, as shown in Fig. 7. Therefore, the greater the nonlinearity of the structure, the more frequently chaotic motion will occur. Meanwhile, for $K_{n l}=0.01$, the system behaves non-chaotically at $K_{1}=0.25$ and 0.8 , but the behavior becomes chaotic for $K_{n l}=0.05$ at all values of $K_{1}$. The results are verified by the values of MLE, which are -0.16 and -0.01 for $K_{1}=0.25$ and 0.8 when $K_{n l}=0.01$ but 0.2 and 0.05 for $K_{1}=0.25$ and 0.8 when $K_{n l}=0.05$, respectively, as shown in Fig. 7(b).

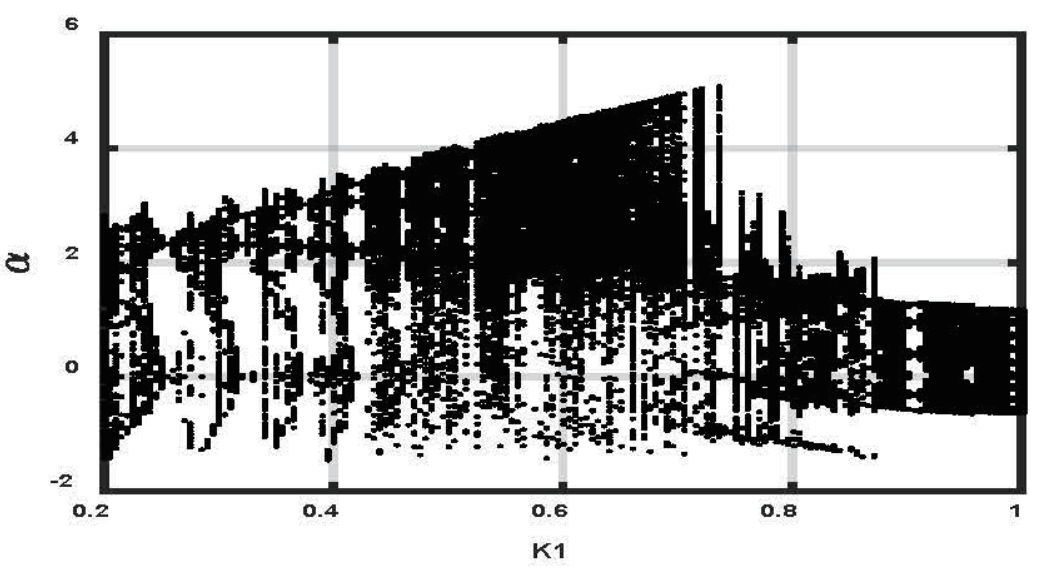

(a)

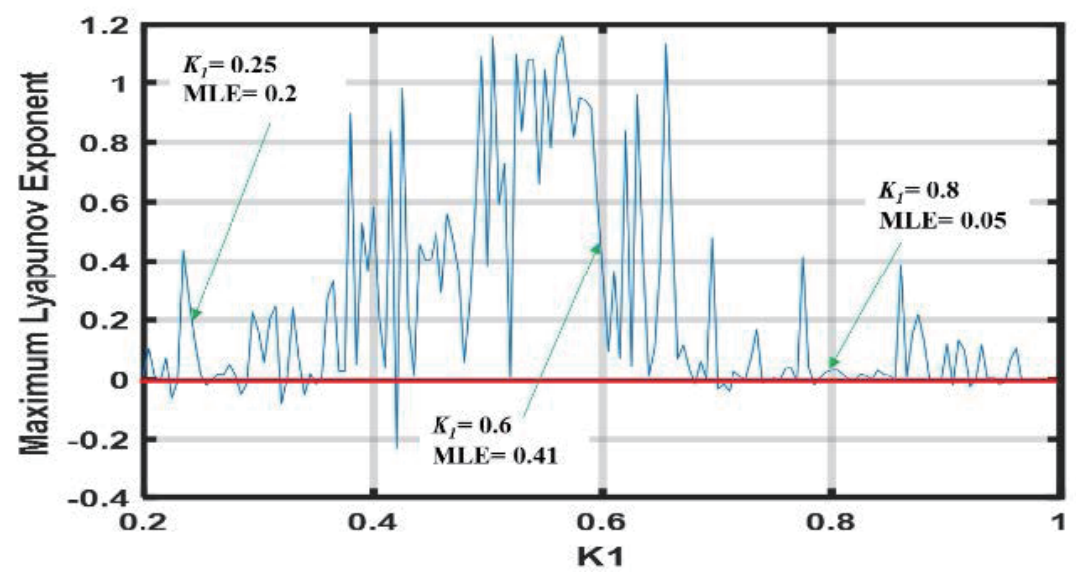

(b)

Fig. 7. (Color online) (a) Bifurcation diagram and (b) MLE for $U=7$ and $K_{n l}=0.05$. Frequency of oscillation is used as an analysis parameter. 


\subsubsection{Influence of airspeed on wind turbine system}

Airspeed is also an important factor determining the performance of wind turbine systems and is correlated with the structural nonlinearity of airfoils. As shown in Fig. 8, when the airspeed is increased to $U=10$ with $K_{n l}=0.01$, the motion is mainly chaotic over the range of $0.2 \leq K_{1}<0.8$. Under the same structural nonlinearity, $K_{n l}=0.01$, chaos occurs for different airspeeds including $U=7$ and 10, and the range of parameters for stable motion of the wind turbine system is $0.8 \leq K_{1} \leq 1.0$, as shown in Figs. 6(b) and 8(b). However, in this range, chaos occurs at $K_{1}=0.92$ and 0.862 when $U=7$ and 10 , respectively. Thus, the results provide conditions to be avoided when designing wind turbine systems.

When the airspeed is increased to $U=10$ with $K_{n l}$ increased to 0.05 , the interval in which chaotic motion occurs expands and stable motion only occurs at specific values of $K_{1}$, as shown in Fig. 9. The pink circles marked in Fig. 9(b) represent stable motion and suggest that the structural nonlinearity of the airfoil induces nonlinear and unstable phenomena. Also, for larger structural nonlinearity $\left(K_{n l}=0.05\right)$ with different airspeeds, as shown in Figs. $7(\mathrm{~b})$ and $9(\mathrm{~b})$, the

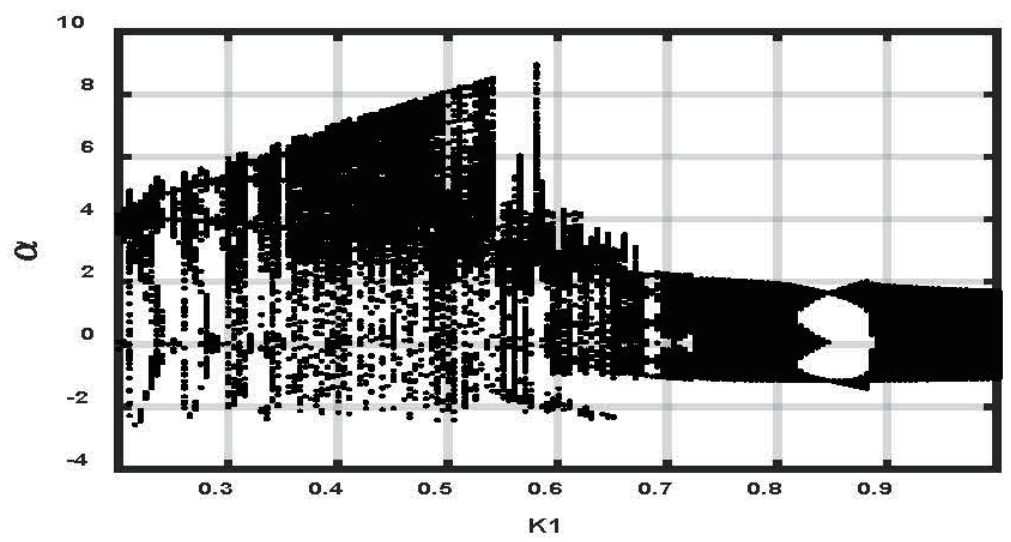

(a)

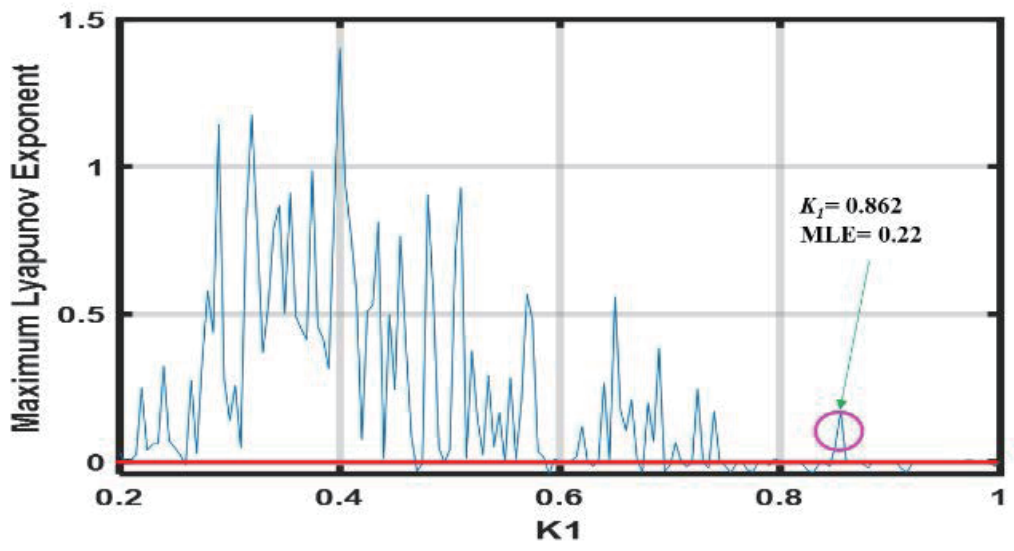

(b)

Fig. 8. (Color online) (a) Bifurcation diagram and (b) MLE for $U=10$ and $K_{n l}=0.01$. Frequency of oscillation is used as an analysis parameter. 


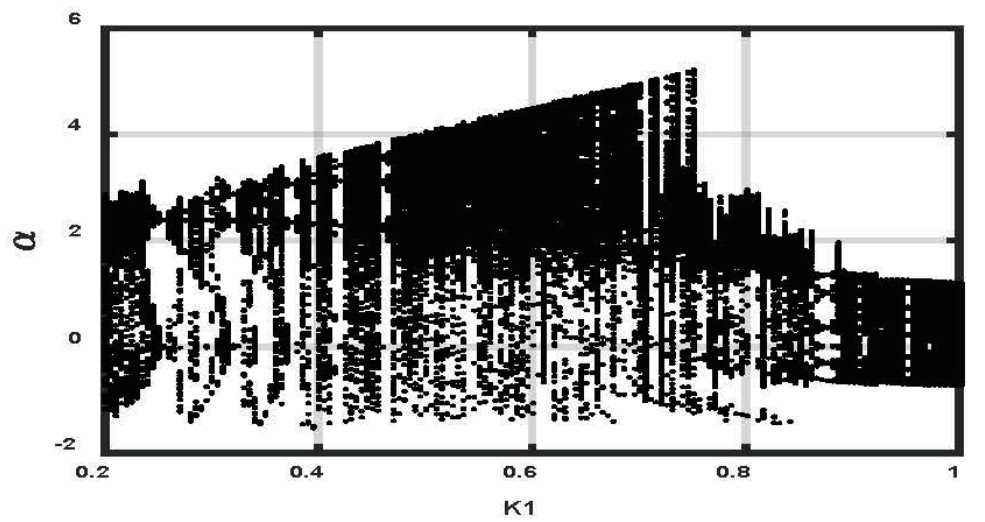

(a)

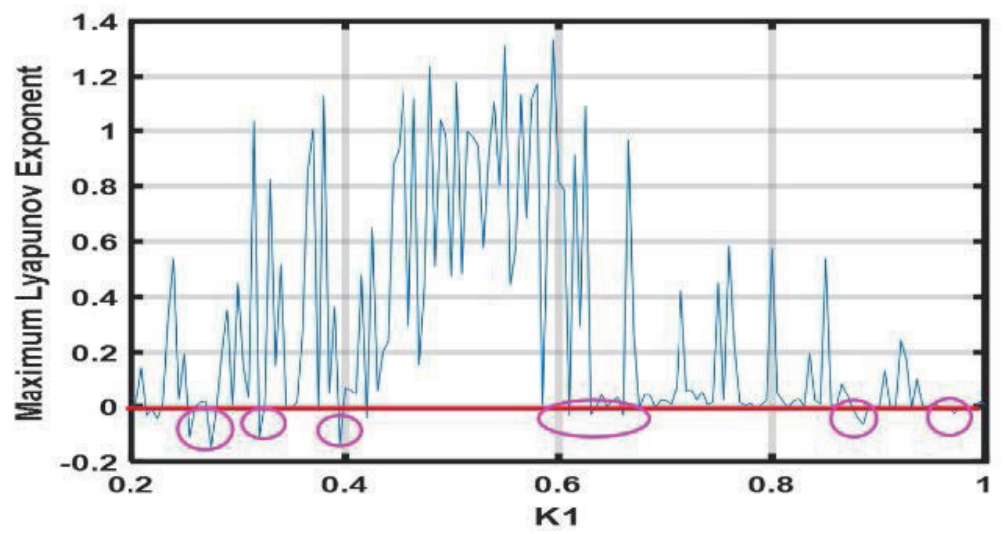

(b)

Fig. 9. (Color online) (a) Bifurcation diagram and (b) MLE for $U=10$ and $K_{n l}=0.05$. Frequency of oscillation is used as an analysis parameter.

wind turbine system more often exhibits chaotic motion than for a lower $K_{n l}$ over the range of $0.2 \leq K_{1} \leq 1.0$ and may even generate unexpected damage.

\section{Conclusion}

In this study, we analyzed the important parameters of a wind turbine airfoil, including the nonlinearity of the structural material, the airspeed, and the reduced frequency of oscillation, and their effects on dynamic stall. This type of analysis can be helpful for industries to find different ways of minimizing dynamic stall. The results show that subharmonic, quasi-periodic, and chaotic motions of the effective angle of attack occur as the reduced frequency of oscillation is changed. Regarding the influence of structural nonlinearity on the wind turbine system, when the structural nonlinearity $\left(K_{n l}\right)$ is increased to 0.05 under the same airspeed $(U)$, chaotic behavior occurs more often than when $K_{n l}=0.01$ in the range of $0.2 \leq K_{1} \leq 1.0$. Therefore, the greater the nonlinearity of the structure, the more frequently chaotic motion will occur. Regarding the influence of the airspeed on the wind turbine system, the occurrence of chaos is 
similar for different airspeeds including $U=7$ and 10, and the range of $K_{1}$ for which the wind turbine system is stable is similar $\left(0.8 \leq K_{1} \leq 1.0\right)$ under the same structural nonlinearity of $K_{n l}=0.01$. For a larger structural nonlinearity $\left(K_{n l}=0.05\right)$ with different airspeeds, the wind turbine system more often generates chaotic motion than at a lower $K_{n l}$ over the range of $0.2 \leq K_{1} \leq 1.0$ and may even generate unexpected damage. Thus, the results of this study provide conditions to be avoided to avoid chaotic motion when designing wind turbine systems.

\section{Acknowledgments}

This research was funded by the Ministry of Science and Technology of Taiwan under grant numbers MOST 109-2221-E-167-007, 110-2221-E-167-019, and 110-2622-E-167-008.

\section{References}

1 N. A. Agbayani: Structures Congr. (2014) 1759-1770. https://doi.org/10.1061/9780784413357.155

2 D. Ancona and J. McVeigh: Wind Turbine-materials and Manufacturing Fact Sheet, Princeton Energy Resources International (2001) http://citeseerx.ist.psu.edu/viewdoc/download?doi=10.1.1.464.5842\&rep=repl\&type=pdf (accessed August 2001).

3 J. Zhong, J. Li, P. Guo, and Y. Wang: Energy 174 (2019) 246. https://doi.org/10.1016/j.energy.2019.02.176

4 C. Zhu, Y. Qiu, and T. Wang: Energy 222 (2021) 120004. https://doi.org/10.1016/j.energy.2021.120004

5 X. Huang, M. Albers, P. S. Meysonnat, M. Meinke, and W. Schroder: Int. J. Heat Fluid Flow 85 (2020) 108668. https://doi.org/10.1016/j.ijheatfluidflow.2020.108668

6 C. Zhu, J. Chen, J. Wu, and T. Wang: Energy 189 (2019) 116272. https://doi.org/10.1016/j.energy.2019.116272

7 X. Liu, S. Liang, G. Li, A. Godbole, and C. Lu: Renewable Energy 156 (2020) 117. https://doi.org/10.1016/j. renene.2020.04.040

8 Q. Wang and Q. Zhao: Aerosp. Sci. Technol. 72 (2018) 502. https://doi.org/10.1016/J.AST.2017.11.033

9 J. Niu, J. Lei, and T. Lu: Aerosp. Sci. Technol. 72 (2018) 476. https://doi.org/10.1016/j.ast.2017.11.030

10 Q. Zhao, Y. Ma, and G. Zhao: Chin. J. Aeronaut. 30 (2017) 1818. https://doi.org/10.1016/j.cja.2017.08.011

11 M. Sun and S. R. Sheikh: Aerosp. Sci. Technol. 3 (1999) 355. https://doi.org/10.1016/S1270-9638(00)86426-3

12 J A. Ekaterinaris and M. F. Platzer: Prog. Aerosp. Sci. 33 (1997) 759. https://doi.org/10.1016/S03760421(97)00012-2

13 M. H. Akbari and S. J. Price: J. Fluids Struct. 17 (2003) 855. https://doi.org/10.1016/S0889-9746(03)00018-5

14 J. W. Larsen, S. R. K. Nielsen, and S. Krenk: J. Fluids Struct. 23 (2007) 959. https://doi.org/10.1016/j. jfluidstructs.2007.02.005

15 X. Liu and J. Xiang: Chin. J. Aeronaut. 19 (2006) 36. https://doi.org/10.1016/S1000-9361(11)60265-3

16 A. R. Mamouri, E Lakzian, and A. B. Khoshnevis: Ocean Eng. 187 (2019) 106229. https://doi.org/10.1016/j. oceaneng.2019.106229

17 A. S. Muhsen: Nonlinear Dynamic Analysis of Wind Turbine Towers Subject to Design Wind and Seismic Loads, Colorado School of Mines, Ph.D. Thesis (2016).

18 C. C. Wang and Y. Q. Zhu: Symmetry 13 (2021) 1445. https://doi.org/10.3390/sym13081445 